\title{
Genetic mechanisms of bone digestion and nutrient absorption in the bone-eating worm Osedax japonicus inferred from transcriptome and gene expression analyses
}

Norio Miyamoto ${ }^{1 *}$, Masa-aki Yoshida ${ }^{2,3}$, Hiroyuki Koga $a^{3,4}$ and Yoshihiro Fujiwara ${ }^{1}$

\begin{abstract}
Background: Bone-eating worms of the genus Osedax (Annelida, Siboglinidae) have adapted to whale fall environments by acquiring a novel characteristic called the root, which branches and penetrates into sunken bones. The worms lack a digestive tract and mouth opening, and it has been suggested that Osedax degrade vertebrate bones and uptake nutrients through acidification and secretion of enzymes from the root. Symbiotic bacteria in the root tissue may have a crucial role in the metabolism of Osedax. However, the molecular mechanisms and cells responsible for bone digestion and nutrient uptake are still unclear, and information on the metabolic interaction between Osedax and symbiotic bacteria is limited.

Results: We compared transcriptomes from three different RNA samples from the following tissues: trunk + palps, root + ovisac, and larva + male. A Pfam domain enrichment analysis revealed that protease- and transporter-related genes were enriched in the root + ovisac specific genes compared with the total transcriptome. Through targeted gene annotation we found gene family expansions resulting in a remarkably large number of matrix metalloproteinase ( $\mathrm{mmp}$ ) genes in the Osedax compared with other invertebrates. Twelve of these Osedax mmp genes were expressed in the root epidermal cells. Genes encoding various types of transporters, including amino acid, oligopeptide, bicarbonate, and sulfate/carboxylate transporters, were also expressed in root epidermal cells. In addition, amino acid and other metabolite transporter genes were expressed in bacteriocytes. These protease and transporter genes were first expressed in root tissues at the juvenile stage, when the root starts to develop.

Conclusions: The expression of various proteinase and transporter genes in the root epidermis supports the theory that the root epidermal cells are responsible for bone digestion and subsequent nutrient uptake. Expression of transporter genes in the host bacteriocytes suggests the presence of metabolic interaction between Osedax and symbiotic bacteria.
\end{abstract}

Keywords: Matrix metalloproteinase, Bone digestion, Solute carrier family transporter, Nutrient uptake

\footnotetext{
*Correspondence: nmiyamoto621@gmail.com

'Japan Agency for Marine-Earth Science and Techonology, Yokosuka,

Kanagawa, Japan

Full list of author information is available at the end of the article
} 


\section{Background}

The deep sea is one of the few remaining frontiers in the field of biology. Since the discovery of an invertebrate community in the Galapagos Rift in 1977 [1], several chemosynthetic ecosystems have been found in hydrothermal vents and hydrocarbon seeps worldwide [2, 3]. In these environments, many endemic species, such as vestimentiferan tubeworms, vesicomyid clams, and Rimicaris shrimps, have been reported [2]. These organisms have evolved to consume new nutrient sources, with chemosynthetic energy obtained through symbiosis with chemosynthetic microbes (reviewed in [4, 5]). In addition to vents and seeps, another type of deep-sea community has also been discovered, referred to as the whale-fall ecosystem [6]. When a carcass of a large vertebrate (e.g., a whale) sinks to the sea floor, the huge source of organic material harbors a variety of organisms. Initially, mobile scavengers such as sharks, hagfishes, and crustaceans aggregate and consume the soft tissue of the carcass [7]. After the bones of the carcass are exposed, enigmatic marine worms of the genus Osedax colonize on the bones [8].

Osedax are marine invertebrates that belong to the phylum Annelida, family Siboglinidae [8]. They exclusively inhabit sunken whale bones under natural conditions and are able to colonize bones of other vertebrate species under experimental conditions [9-11]. Since their discovery, at least 27 higher taxonomic units have been described worldwide [8, 11-19]. Osedax usually shows remarkable sexual dimorphism, with vermiform females (Fig. 1a, b), and microscopic dwarf males (Fig. 1c) [8]. Osedax has trochophore-type larvae (Fig. 1d). Adult females of Osedax consist of four regions: palps, trunk, ovisac, and root. By consuming vertebrate bones, Osedax worms play a role in the degradation of sunken body remains and the recycling of deep-sea carbon $[15,20]$. The female Osedax lacks a digestive tract, including a mouth, gut, and anus. Instead, it has been suggested that they use their posterior root system as their digestive organ. The posteriorly branching root system is an evolutionary novel organ $[8,20,21]$, which penetrates into bones and contains heterotrophic bacteria enclosed in bacteriocytes (Fig. 1a, b). It has been suggested that the microvillar root cells secrete digestive enzymes and acid to aid in the degradation and uptake of nutrients from the dissolved bones [22-25]. Although it has been assumed that these endosymbiotic bacteria have a function in the metabolism of nutrients, the specific process remains unclear [20, 23, 26, 27]. To address the evolution of Osedax worms, an understanding of the origin and detailed function of the root is necessary. A recent study has shown that the root epidermal cells are immunoreactive against anti-vascular proton ATPase $\left(\mathrm{V}-\mathrm{H}^{+}\right.$ATPase) and anti-carbonic anhydrase (CA) antibodies [24]. This suggests that $\mathrm{V}-\mathrm{H}^{+}$ATPase are responsible for dissolving the calcium phosphate in vertebrate bones through acidification of the microenvironment surrounding the root [24]. Another study, which showed that the root of Osedax has collagenolytic activity, suggested that the root tissues that include symbiotic bacteria secrete enzymes to digest the matrix proteins of bones [23]. The absence of matrix proteinase gene in the symbiont genomes suggests that host worms are responsible for collagen degradation [27]. However, it is not known what varieties of enzymes are secreted from what type of cells, and the mechanisms behind nutrient uptake.

Here, we performed transcriptome analysis and examined the spatial and developmental expression patterns of genes related to digestion and nutrient uptake in the bone-eating worm Osedax japonicus, which can be reared for multiple generations under laboratory conditions [28]. Comparing the transcriptomes from the three different tissues: root + ovisac (root), trunk + palp (trunk), and larva + male, we identified the genes that were specifically expressed in the root. We investigated the expression patterns of genes of interest that were considered related to bone digestion and nutrient uptake. The results revealed novel aspects of the molecular mechanisms of the metabolic process of Osedax worms.
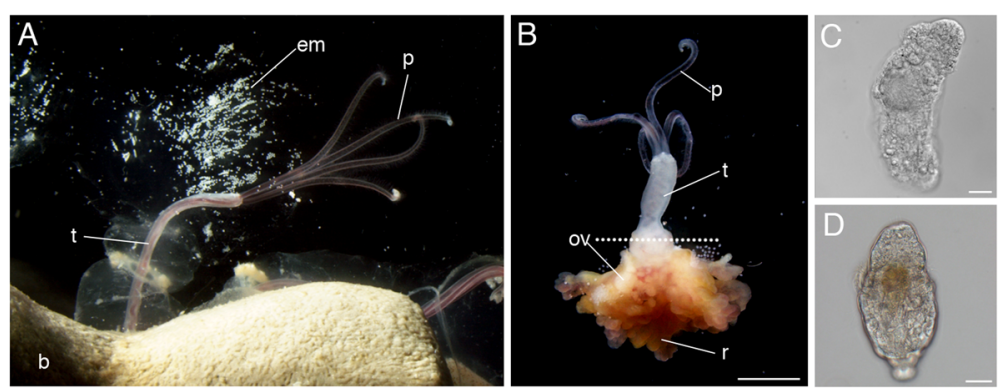

Fig. 1 Photographs Osedax japonicus. a An adult female living on a bone. White spots around the female are embryos spawned into transparent mucus. $\mathbf{b}$ An adult female exposed on a bone. The dot line indicates the position that was cut for RNA extraction. Upper and lower fragments are trunk palps (trunk) and root + ovisac (root), respectively. c A dwarf male. d A larva with settlement competency. b, bone; em, embryo; ov, ovisac; p, palp; r, root; t, trunk. Scale bars: $B=2 \mathrm{~mm} ; C$ and $D=20 \mu \mathrm{m}$ 


\section{Results}

\section{Sequencing of $O$. japonicus transcriptome}

We sequenced three types of RNA samples: trunk, root, and larva + male (Fig. 1a-d) using a HiSeq2000 sequencer (Illumina, San Diego, CA, USA). Males of O. japonicus are dwarf males and are approximately $500 \mu \mathrm{m}$ in length (Fig. 1c). Owing to the small amounts of total RNA obtained from males and larvae, these two sample types were combined for sequencing. A total of 200,523,188 reads were obtained and the basic information regarding the raw data and assembly is shown in the supporting information (Additional file 1: Figure S1, Additional file 2: Table S1). The raw reads were assembled into 57,194 contigs and 38,913 subcomponents by Trinity software [29] with a contig N50 of $1383 \mathrm{bp}$. Although the read assembly was still incomplete, we considered these subcomponents to be distinct genetic loci. We set the threshold for gene expression intensity (fragments per kilobase per million reads, $[F P K M] \geq 1$, see Methods) to eliminate fluctuations or background noise and 22,541 genes were found above the threshold in at least one sample. Among 22,541 genes, the numbers of trunk, root, and larva + male-specific genes were 825,1214 , and 5485 , respectively. The 22,541 genes were compared against UniprotKB with BLASTX and 9448 genes were homologous to known functional genes. Among these 9448 genes, 185, 223, and 1258 genes were trunk, root, and larva + male specific, respectively. The list of root specific gene is shown in the supporting information (Additional file 3: Figure S2, Additional file 4: Table S2). Raw data are available in the DDBJ sequence Read Archive (DRA003880).

\section{Characterization of the root transcriptome}

To elucidate the features of O. japonicus transcriptome, we attempted functional annotation of novel sequence data using gene ontology (GO) annotation and a single enrichment analysis against the total $O$. japonicas transcriptome. GO term enrichment analysis showed that 11, nine, and six terms associated with "molecular function," "cellular component," and "biological process," respectively, were significantly over-represented in the root-specific genes against all gene set backgrounds (Additional file 5: Table S3). The over-represented GO terms included hydrolase activity, transferase activity, transport, and catalytic activity, which were all related to the nutrient uptake and metabolic processes. Only two terms of "molecular function" and a term of "biological process" were under-represented in the root transcriptome (Additional file 5: Table S3).

We also looked for significantly enriched Pfam domains [30] in the proteins encoded by the root-specific genes against the total transcriptome (Table 1). In the root-specific genes, we found 36 enriched Pfam domains (Table 1). Matrixin (PF00413), hemopexin (PF00045), putative peptidoglycan binding domain (PF01471), and peptidase families (PF05649, PF01433, and PF01431) are components of matrix metalloproteinase (MMP) proteins, which degrade the extracellular matrix (ECM) including various types of collagen. Pfam domains related to other types of proteases were also enriched in the root-specific gene set. The peptidase family M13 (PF05649) is a domain found in membrane metalloendopeptidase (MME), which cleaves peptides at the amino side of hydrophobic residues. Zinc carboxypeptidase (PF00246) and carboxypeptidase activation peptide (PF02244) are domains related to carboxypeptidase activity that hydrolyze peptides at the carboxyl-terminal end of proteins and peptides. We also found an enrichment of domains related to substrate transportation. The amino acid permeases (PF13520 and PF00324) domain is a component of amino acid transporters belonging to the solute carrier (SLC) family transporters, such as the SLC7 and SLC12 families. We found that three domains related to another member of the SLC transporter family were enriched in the root transcriptome. Sodium:sulfate symporter transmembrane region (PF00939) and citrate transporter (PF03600) are components of the SLC13 family $\mathrm{Na}^{+}$-sulfate/carboxylate cotransporter. The domain related to a sugar transporter (PF00083) was also enriched in the root-specific gene set. The ABC transporter transmembrane region (PF00664) and $\mathrm{ABC}$ transporter (PF00005) are domains related to the ATP-binding cassette $(\mathrm{ABC})$ transporter, which translocates a variety of substrates.

\section{Expression of protease genes in the root}

To identify cells responsible for protease secretion, we examined expression pattern of genes encoding protease, which degraded the ECM. Because Pfam domains related to the MMP protein were enriched in the root-specific gene set, we first examined the expression of $m m p$ genes. From the transcriptome of all samples, $24 \mathrm{mmp}$ genes containing the catalytic domain with a conserved zincbinding motif were found. Among them, $22 \mathrm{mmp}$ genes were detected from the root transcriptome and $13 \mathrm{mmp}$ genes were expressed only in the root (Additional file 6, Table S4). We examined the expression of $16 \mathrm{mmp}$ genes and performed double staining with a probe against the $16 S$ rRNA gene of Neptunomonas japonica (Nj $16 S$ rRNA) infected with $O$. japonicus to clarify the localization of bacteriocytes harboring symbiotic bacteria.

Eight of the $16 \mathrm{mmp}$ genes were solely expressed in the epidermis of the root (Fig. 2a-j): Oja-mmp5, Oja-mmp6, Oja-mmp7, Oja-mmp15, Oja-mmp17, Oja-mmp20, Oja$m m p 21$, and Oja-mmp24. The details of the expression patterns in these genes differed. Oja-mmp21 was most broadly expressed (Fig. 2a-c). Counterstaining with 4',6-diamino-2-2phenylindole (DAPI) showed that Oja$m m p 21$ was exclusively expressed in epidermal cells 
Table 1 Pfam domains enriched in the root transcriptome

\begin{tabular}{|c|c|c|c|c|c|}
\hline Pfam ID & Domain description & Total & Root & P.value & Q.value \\
\hline PF00413.19 & Matrixin & 62 & 26 & $5.96 \mathrm{E}-22$ & $8.10 \mathrm{E}-20$ \\
\hline PF00045.14 & Hemopexin & 40 & 20 & 4.45E-19 & $3.02 \mathrm{E}-17$ \\
\hline PF01471.13 & Putative peptidoglycan binding domain & 31 & 14 & $5.95 \mathrm{E}-13$ & 2.69E-11 \\
\hline PF05649.8 & Peptidase family M13 & 34 & 12 & $8.85 \mathrm{E}-10$ & 3.01E-08 \\
\hline PF13520.1 & Amino acid permease & 21 & 7 & 4.94E-06 & $1.12 \mathrm{E}-04$ \\
\hline PF07690.11 & Major Facilitator Superfamily & 123 & 16 & $6.63 \mathrm{E}-06$ & 1.29E-04 \\
\hline PF00939.14 & Sodium:sulfate symporter transmembrane region & 10 & 5 & 1.17E-05 & 1.76E-04 \\
\hline PF03600.11 & Citrate transporter & 10 & 5 & 1.17E-05 & 1.76E-04 \\
\hline PF00324.16 & Amino acid permease & 24 & 7 & $1.34 \mathrm{E}-05$ & 1.82E-04 \\
\hline PF00246.19 & Zinc carboxypeptidase & 27 & 7 & $3.14 \mathrm{E}-05$ & 3.88E-04 \\
\hline PF07953.7 & Clostridium neurotoxin, $\mathrm{N}$-terminal receptor binding & 7 & 4 & 4.93E-05 & 5.15E-04 \\
\hline PF12388.3 & Dual-action HEIGH metallo-peptidase & 7 & 4 & 4.93E-05 & 5.15E-04 \\
\hline PF14670.1 & Coagulation Factor Xa inhibitory site & 68 & 10 & $1.27 \mathrm{E}-05$ & $1.23 \mathrm{E}-03$ \\
\hline PF01433.15 & Peptidase family M1 & 24 & 6 & 1.47E-04 & 1.33E-03 \\
\hline PF14653.1 & Insulin growth factor-like family & 16 & 5 & $1.70 \mathrm{E}-04$ & $1.44 \mathrm{E}-03$ \\
\hline PF01431.16 & Peptidase family M13 & 36 & 7 & $2.24 \mathrm{E}-04$ & $1.79 \mathrm{E}-03$ \\
\hline PF02244.11 & Carboxypeptidase activation peptide & 6 & 3 & 8.07E-04 & 5.77E-03 \\
\hline PF00431.15 & CUB domain & 46 & 7 & 1.06E-03 & 7.20E-03 \\
\hline PF05572.8 & Pregnancy-associated plasma protein-A & 7 & 3 & 1.37E-03 & $8.90 \mathrm{E}-03$ \\
\hline PF01607.19 & Chitin binding Peritrophin-A domain & 63 & 8 & $1.60 \mathrm{E}-03$ & $9.88 \mathrm{E}-03$ \\
\hline PF13582.1 & Metallo-peptidase family M12B Reprolysin-like & 27 & 5 & 2.27E-03 & $1.29 \mathrm{E}-02$ \\
\hline PF00664.18 & $\mathrm{ABC}$ transporter transmembrane region & 41 & 6 & 2.95E-03 & $1.53 \mathrm{E}-02$ \\
\hline PF10462.4 & Peptidase M66 & 9 & 3 & $3.13 \mathrm{E}-03$ & 1.53E-02 \\
\hline PF00209.13 & Sodium:neurotransmitter symporter family & 32 & 5 & 4.91E-03 & $2.22 \mathrm{E}-02$ \\
\hline PF13385.1 & Concanavalin A-like lectin/glucanases superfamily & 80 & 8 & 7.12E-03 & 2.93E-02 \\
\hline PF00005.22 & $\mathrm{ABC}$ transporter & 66 & 7 & $8.43 \mathrm{E}-03$ & 3.37E-02 \\
\hline PF13688.1 & Metallo-peptidase family M12 & 24 & 4 & 9.29E-03 & $3.58 \mathrm{E}-02$ \\
\hline PF01391.13 & Collagen triple helix repeat (20 copies) & 39 & 5 & 1.15E-02 & $3.58 \mathrm{E}-02$ \\
\hline PF03209.10 & PUCC protein & 5 & 2 & 1.16E-02 & $3.58 \mathrm{E}-02$ \\
\hline PF04727.8 & ELMO/CED-12 family & 5 & 2 & 1.16E-02 & $3.58 \mathrm{E}-02$ \\
\hline PF11669.3 & WW domain-binding protein 1 & 5 & 2 & 1.16E-02 & $3.58 \mathrm{E}-02$ \\
\hline PF13243.1 & Prenyltransferase-like & 5 & 2 & 1.16E-02 & $3.58 \mathrm{E}-02$ \\
\hline PF11838.3 & ERAP1-like C-terminal domain & 14 & 3 & 1.19E-02 & $3.58 \mathrm{E}-02$ \\
\hline PF00629.18 & MAM domain & 26 & 4 & $1.24 \mathrm{E}-02$ & $3.58 \mathrm{E}-02$ \\
\hline PF00012.15 & Hsp70 protein & 15 & 3 & $1.45 \mathrm{E}-02$ & 4.10E-02 \\
\hline PF00083.19 & Sugar (and other) transporter & 43 & 5 & $1.71 \mathrm{E}-02$ & 4.65E-02 \\
\hline
\end{tabular}

(Fig. 2c). With the exception of Oja-mmp17, all of these genes were specific to the root transcriptome (Additional file 7: Table S4) and positive signals were detected only in the peripheral branching region of the root and not in the epidermis of the ovisac. Doublestaining with $N j 16 S$ rRNA indicated that five $m m p$ genes, Oja-mmp12, Oja-mmp16, Oja-mmp18, Oja-mmp19, and Oja-mmp22 were expressed in bacteriocytes (Fig. 2k-o) with all but Oja$m m p 22$ also expressed in the root epidermis (Fig. $2 \mathrm{k}-\mathrm{n}$ ).
Three genes, Oja-mmp9, Oja-mmp10, and Oja-mmp11 were solely expressed in the internal tissues related to the reproductive system. The expression of Oja-mmp9 and Oja-mmp10 were detected in the epithelial cells of ovarian tissue (Fig. 2p, q). Expression of Oja-mmp9 was detected only in the epithelial cells of the uterus [31] surrounding mature oocytes. Counterstaining with DAPI showed that Oja-mmp10 was also expressed in connective tissues around the uterus (Fig. 2r, s). In O. japonicus, the ovarian 


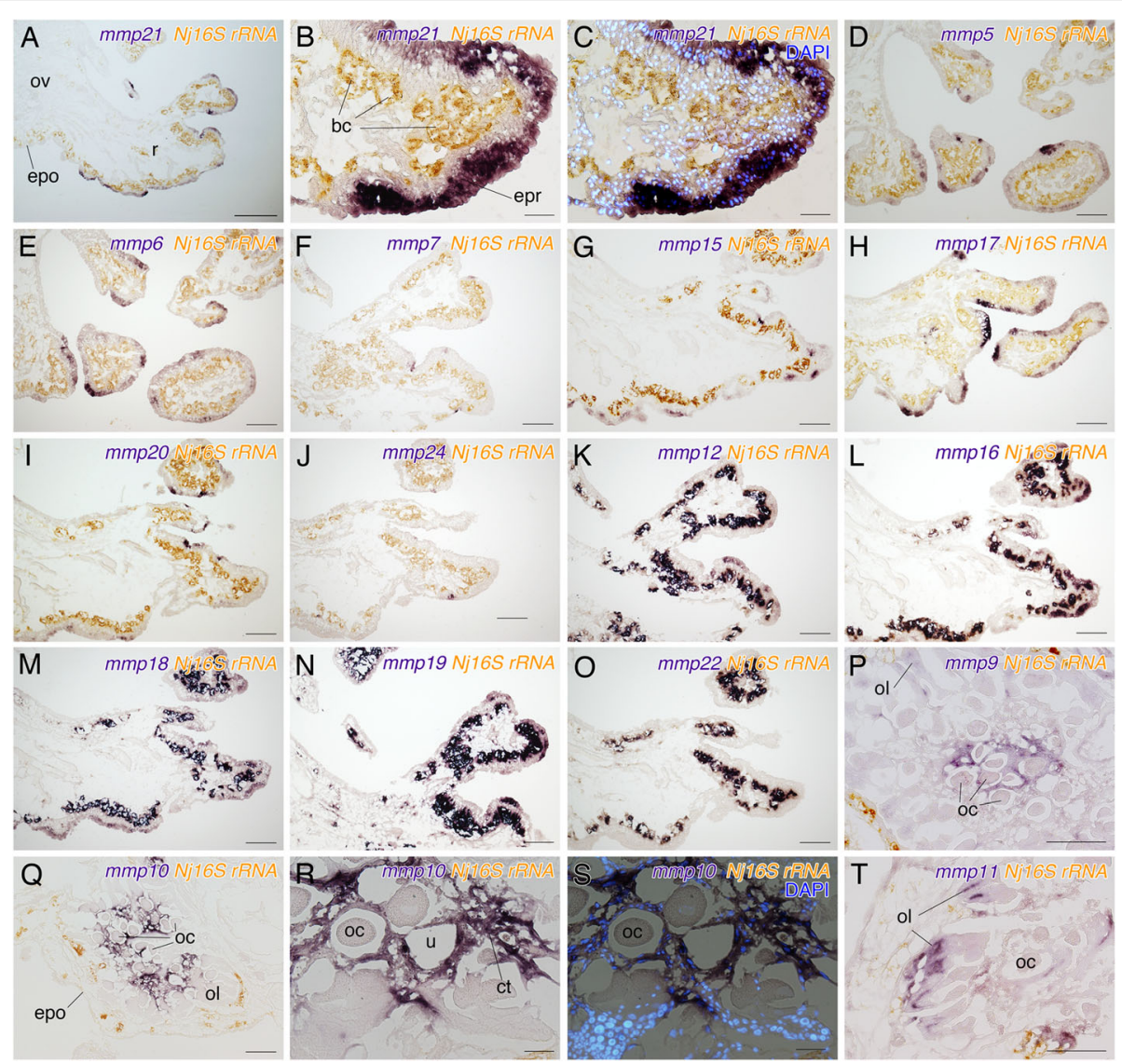

Fig. 2 Expression patterns of $m m p$ genes in Osedax japonicus. Purple signals are expression of mmp genes and yellow signals are expression of 165 rRNA of Neptunomonas japonica. Sagittal sections. Anterior is top. a-c Broad epidermal expression of Oja-mmp21. d-j Expression patterns of Oja-mmp5, Oja-mmp6, Oja-mmp7, Oja-mmp15, Oja-mmp17, Oja-mmp20, and Oja-mmp24 in the epidermis of the root. $\mathbf{k}-\mathbf{n}$ Expression patterns of Oja-mmp12, Oja-mmp16, Oja-mmp18, and Oja-mmp19 in the root epidermis and bacteriocytes. o Expression of Oja-mmp22 in bacteriocytes. $\mathbf{p}$ Expression of Oja-mmp9 in follicle cells and connective tissue surrounding oocytes. q-s Expression of Oja-mmp10 was detected in the connective tissue surrounding oocytes. Counterstaining with DAPI indicated that Oja-mmp10 was also expressed in follicle cells. $\mathbf{t}$ Oja-mmp 11 expression detected in the germinal epithelium between the stalks of developing oocytes. bc, bacteriocyte; ct, connective tissue; epo, ovisac

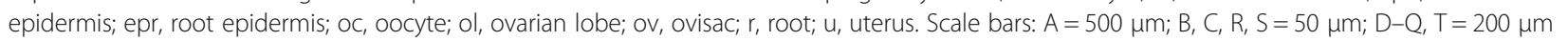

lobe consists of two rows of previtellogenic oocytes. Between the two rows of previtellogenic oocytes there are thin cells and Oja-mmp11 expression was observed in these cells (Fig. 2t).

Other varieties of proteases were also found in the root transcriptome. Three cathepsin Bs (ctsB genes: OjactsB1, Oja-ctsB2, and Oja-ctsB3) were identified in Osedax. Oja-ctsB2 and Oja-ctsB3 were present exclusively in the root transcriptome, whereas Oja-ctsB1 was detected in all three transcriptomes (Additional file 6: Table S4). The expressions of Oja-ctsB2 and Oja-ctsB3 were detected in the root epidermis (Fig. $3 a-c)$. The expression patterns of Oja-ctsB2 and Oja-ctsB3 were almost identical, with both genes expressed in the epidermis of the distal part of the branching root. No signal was detected at the proximal part, which is close to the ovaries. The expressions of Oja-ctsB2 and Oja-ctsB3 were not ubiquitous in root epidermis. There were negative cells in the distal part of the root, which were thin, whereas the positive regions were thick (Fig. 3a-c). MME is a type II transmembrane glycoprotein and a neutral endopeptidase that belongs to the peptidase family M13. As mentioned above, the peptidase family M13 domain was enriched in the root transcriptome (Table 1). An mme gene, Oja-mme was present only in the root transcriptome (Additional file 6: Table S4) and expressed in root epidermal cells (Fig. 3d, e).

\section{Expression of transporter genes in the root}

SLC family transporters play crucial roles in the uptake and efflux of compounds such as sugars, amino acids, nucleotides, inorganic ions, and fatty acids [32, 33]. In the O. japonicus transcriptome, 40 families in the $s l c$ gene superfamily, with at least 233 genes, were identified 


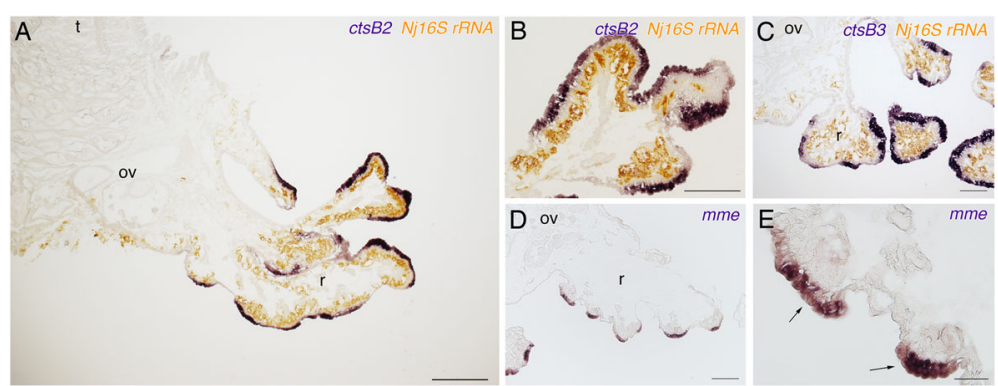

Fig. 3 Expression of protease genes. a, b Expression of Oja-ctsB2 detected in root epidermis. c Oja-ctsB3 expressed in root epidermis. d, e Oja-mme expressed in root epidermal cells. Positive signals were detected only in thick cells. ov, ovisac; r, root; t, trunk. Scale bars: A =500 $\mu$ m; $B-D=200 \mu m ; E=50 \mu m$

(Additional file 8: Table S5). Although the slc gene nomenclature system has been defined in mammals [33], due to low resolution of the phylogenetic analyses and the likely lineage-specific molecular evolution of the $s l c$ genes, the genes were named in an Osedax-specific manner.

The SLC15 family members are proton-coupled oligopeptide transporters in vertebrates [34]. In the $O$. japonicus root transcriptome, two genes of the SLC15 family, Oja-slc15a-1 and Oja-slc15a-2, were found. Oja-slc15a-2 was detected only from the root transcriptome. These two genes were expressed in the epidermis of the root (Fig. 4a-g). In the root, expression was restricted to the peripheral branching regions and no signal was detected in the epidermis of the ovisac (Fig. 4a). In the distal part of the root, there were some cells in which a positive signal was not detected (Fig. 4c, f, arrowheads). A nuclear counter stain with DAPI showed that these genes were expressed exclusively in the epidermis (Fig. $4 \mathrm{~d}, \mathrm{~g}$ ). The members of the SLC6 family are $\mathrm{Na}^{+}$- and $\mathrm{Cl}^{-}$-dependent neurotransmitter symporters that translocate small amino acid or amino acid-like substrates [35]. Expression of Oja-slc6a-1 was detected in bacteriocytes where it overlapped a positive $N j 16 S r R N A$ signal (Fig. 4h). Oja-slc6a-2 was also expressed in the root epidermis (Fig. 4i). Expression was not widespread in the epidermis, being restricted to small populations of epidermal cells (Fig. 4i). Transporters of the SLC7 family are amino acid transporters [36]. In O. japonicus, a slc7 gene, Oja-slc7a-1, was expressed in bacteriocytes (Fig. 4j). The SLC17 family has been described as vesicular glutamate transporters [37]. In O. japonicus, a positive Oja-slc17a-1 signal overlapped with that of $\mathrm{Nj} 16 \mathrm{~S} r R N A$. This expression indicated that Oja-slc17a-1 was expressed in bacteriocytes (Fig. 4k).

The expression patterns of other metabolite transporters were also examined. The members of the SLC5 family are glucose transporters that play an important role in sugar uptake [38]. In O. japonicus, Oja-slc5a-1 was expressed in the bacteriocytes (Fig. 4l). The SLC16 family members are monocarboxylate transporters that catalyze the proton- linked transport of monocarboxylates such as lactate, pyruvate and ketone bodies [39]. A member of the slc16 family gene, Oja-slc16a-1, was expressed in the bacteriocytes (Fig. 4m). The SLC13 family members are $\mathrm{Na}^{+}$and $\mathrm{SO}_{4}^{2-}$-carboxylate co-transporters, which translocate anions, or di- and tri-carboxylate citric acid cycle intermediates in vertebrates [40]. Two slc13 genes, Oja-slc13a-1 and Oja-slc13a-2, were expressed in the root epidermis (Fig. 4n, o). Expression of Oja-slc13a-1 was widespread in root epidermis (Fig. 4n), whereas Oja-slc13a-2 was expressed only in a small number of root epidermal cells (Fig. 4o, arrowheads). The SLC4 family of bicarbonate transporters transfers the bicarbonate ion or a related species across the plasma membrane [41]. Oja-slc4a-1 was expressed in the mesodermal cells in the root (Fig. 4p). A nucleus counterstain with DAPI detected positive Ojaslc4a-1 signals in the cell layer just underneath the epidermis, which was identified as a muscle layer (Fig. 4q, r, [26]). Oja-slc4a-2 was expressed in the root epidermis and non-symbiotic mesenchymal cells around bacteriocytes (Fig. 4s, arrows).

\section{Expression of protease and transporter genes in the larval and juvenile stages}

For the larval stages, expressions of Oja-mmp6, Ojammp12, Oja-mmp18, Oja-ctsB2, Oja-ctsB3, Oja-slc6a-1, Oja-slc6a-2, and Oja-slc15a-2 genes were not detected (Fig. 5a-g, i). Oja-slc15a-1 was expressed in the internal tissue of larvae (Fig. 5h). However all of these genes were expressed in the root tissue at the juvenile stage, 2-5 days after settlement (Fig. 5j-r). Oja-mmp6, Oja-mmp12, Ojammp18, Oja-ctsB3, Oja-slc15a-1, and Oja-slc15a-2 were expressed widely in the root epidermis of juveniles (Fig. 5 , $\mathrm{k}, \mathrm{ln}, \mathrm{q}, \mathrm{r}$ ). Oja-ctsB2 was expressed in the posterior part of the developing root, whereas Oja-ctsB3 was expressed widely in the root (Fig. 5m, n). A positive Oja-slc6a-1 signal was detected inside the root where bacteriocytes were present (Fig. 5o). Oja-slc6a-2 was expressed in part of the root epidermis (Fig. 5p). 


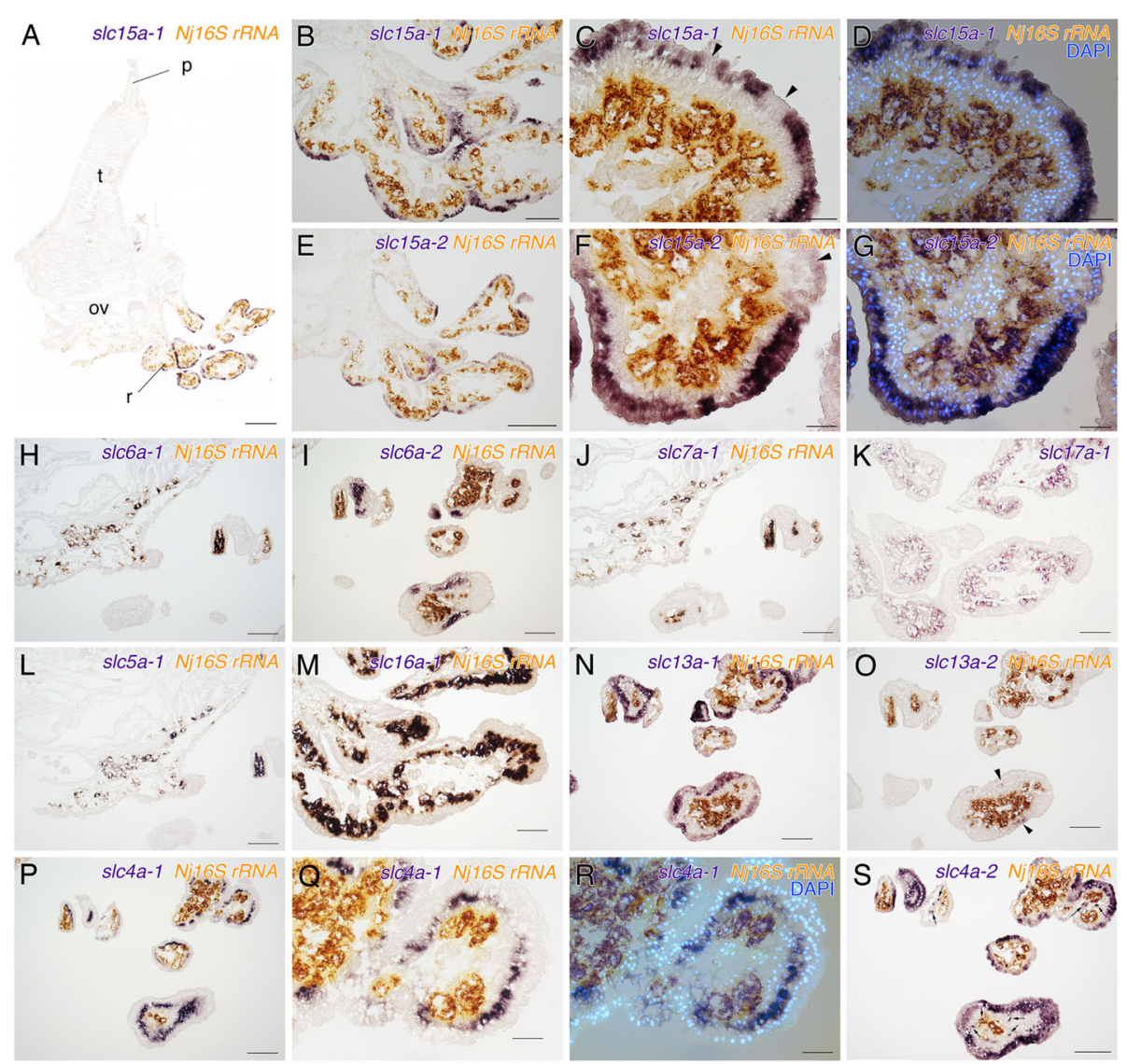

Fig. 4 Expression patterns of s/c genes in Osedax japonicus. a-d Expression of an oligopeptide transporter gene Oja-s/c15a-1. a, b Positive signals detected at the distal part of root tissue, where there is no ovisac beneath the epidermis. $\mathbf{c}$, d Counterstaining with DAPI showed that Oja-s/c15a-1 was expressed only in root epidermal cells. e- $\mathbf{g}$ Expression of another oligopeptide transporter gene Oja-cls 15a-2. f, $\mathbf{g}$ A high-magnification image and counterstaining with DAPI indicated that Oja-s/C15a-2 was expressed in root epidermal cells. $\mathbf{h}$ Double-staining with Nj16S rRNA showed an amino acid transporter gene Oja-s/c6a-1 expressed in bacteriocytes. i An amino acid transporter gene Oja-s/c6a-2 was expressed in root epidermal cells. $\mathbf{j}$ Double-staining with $\mathrm{Nj}$ 165 rRNA showed that an amino acid transporter gene Oja-slc7a-1 was expressed in bacteriocytes. $\mathbf{k}$ Expression of an amino acid transporter gene Oja-s/c17a-1 was detected in bacteriocytes. I Double-staining with Nj 165 rRNA indicated that a glucose transporter gene Oja-s/c5a-1 was expressed in bacteriocytes. $\mathbf{m}$ Double-staining with Nj 165 rRNA indicated that a monocarboxylate transporter gene Oja-s/c16a-1 was expressed in bacteriocytes. n, o Expression of two carboxylate transporter genes, Oja-s/c13a-1 and Oja-s/c13a-2. Expression of Oja-s/c13a-1 was detected broadly in root epidermis, whereas expression of Oja-s/c13a-2 was detected only in restricted cells of the root epidermis (arrowheads). $\mathbf{p}-\mathbf{s}$ Expression patterns of two bicarbonate transporter genes, Oja-s/c4a-1 and Oja-s/c4a-2. q, $\mathbf{r}$ Counterstaining with DAPI indicated that Oja-s/c4a-1 was expressed in mesodermal cells underlying the epidermis of the root. $\mathbf{s}$ Oja-s/c4a-2 expression detected in root epidermal cells. The gene was also expressed in mesenchymal cells in the root (arrow). ov, ovisac; p, palp; r, root; t, trunk. Scale bars: A = $500 \mu \mathrm{m} ; \mathrm{B}, \mathrm{E}, \mathrm{H}-\mathrm{Q}, \mathrm{S}=200 \mu \mathrm{m} ; \mathrm{C}, \mathrm{D}, \mathrm{F}, \mathrm{G}, \mathrm{R}=50 \mu \mathrm{m}$

\section{Discussion}

\section{Bone digestion by proteases}

Vertebrate bones are made of both organic and inorganic components. One of the main inorganic components is calcium phosphate. A previous study reported that Osedax root epidermis cells are immunoreactive against anti-V- $\mathrm{H}^{+}$ATPase and anti-CA antibodies [24]. The $\mathrm{V}-\mathrm{H}^{+}$ATPase and CA are also expressed in the osteoclasts in vertebrates and are involved in the resorption of bones through acidification [42]. Vertebrate bones and cartilage also contain many different organic components, with the majority being ECM proteins, such as various types of collagens. MMPs are a large family of zinc dependent endoproteinases and are able to degrade ECM components [43]. Vertebrates have seven types of fibrillar collagens, encoded by eleven genes [44]. Types I, V, and XXIV are components of mineralized bone, whereas types II, XI, and XXVII are components of cartilage [44]. In this study, we found at least 24 MMPs in $O$. japonicus. Of these genes, 12 were expressed in the root epidermis. The number of $m m p$ genes is small in invertebrates (two in the fly Drosophila melanogaster and seven in the ascidian Ciona intestinalis, [45]), except for in the sea urchin ([46]; 26 in the purple sea urchin Strongylocentrotus purpuratus). In vertebrates, at least 25 different mmp genes have been identified with 24 of them present in humans [47]. In spiralians, little is known about mmp genes. We surveyed the genomes of three spiralians: 


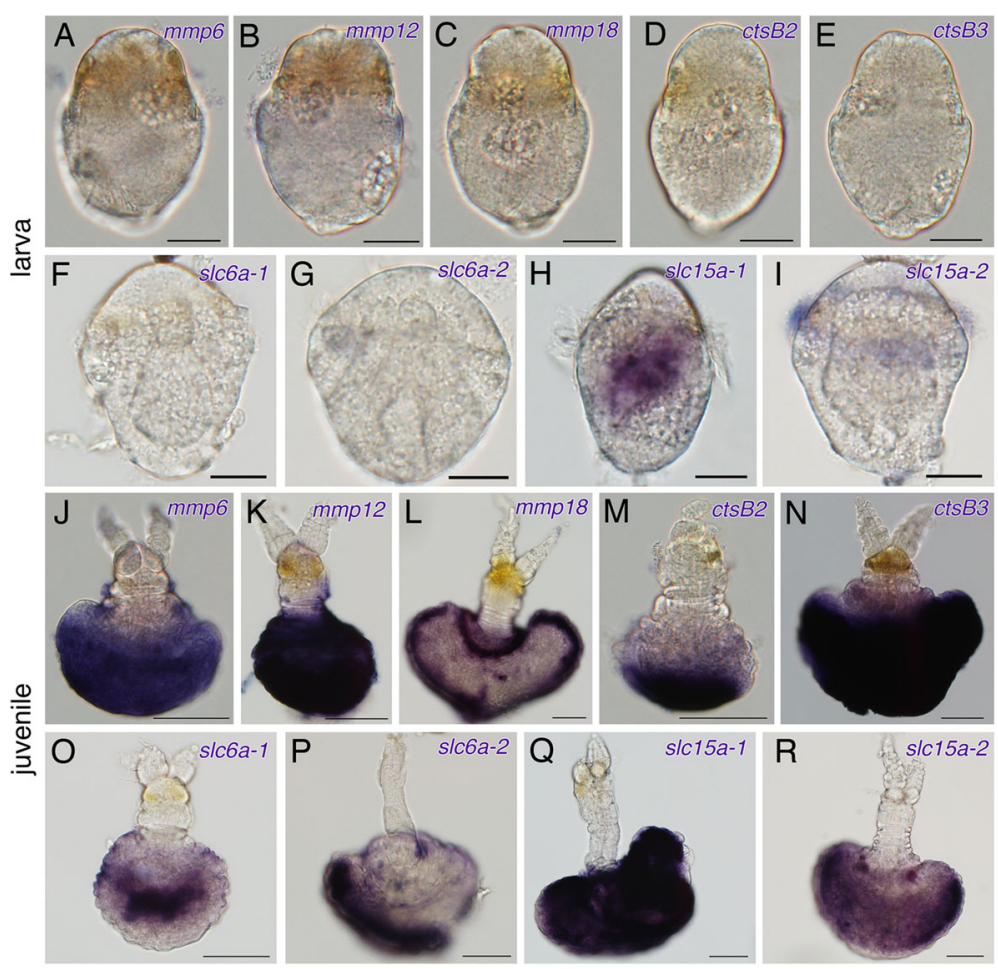

Fig. 5 Expression patterns of protease and transporter genes in larvae and juveniles of Osedax japonicus. Oja-mmp6, Oja-mmp12, Oja-mmp18, Oja-ctsB2, Oja-ctsB3, Oja-s/c6a-1, Oja-s/c6a-2, Oja-s/c15a-1 and Oja-s/c15a-2 expression in larvae (a-i) and juveniles (j-r). At the larval stage, only expression of Oja-s/c15a-1 was detected in the endoderm. j-I Three mmp genes, Oja-mmp6, Oja-mmp12, and Oja-mmp18 were expressed in the root epidermal cells. $\mathbf{m}, \mathbf{n}$ Expression of Oja-ctsB2 was detected in the posterior part of the root epidermis, whereas expression of Oja-ctsB3 was detected widely in root epidermal cells. o Expression of Oja-s/c6a-1 was detected inside cells of the root, where bacteriocytes were located. $\mathbf{p}$ Oja-s/c6a-2 expression was detected in part of the root epidermis. q, $\mathbf{r}$ Expression of Oja-s/c15a-1 and Oja-s/c15a-2 were detected in root epidermal cells. Scale bars $=50 \mu \mathrm{m}$

the pacific oyster Crassostrea gigas [48], the owl limpet Lottia gigantea, and a marine polychaete Capitella teleta [49], and found six, eight and five $m m p$ genes, respectively.

This remarkable increase of MMPs followed by amino acid substitutions and expression in the root epidermis in O. japonicus most likely enables them to digest various types of ECMs in vertebrate bones. In addition to ECM degradation in bone and cartilage, MMPs play essential roles in morphogenesis, immune system functioning, and reproduction through the remodeling of ECMs in vertebrates and invertebrates [50,51]. We detected expression of some Osedax mmp genes in root epidermis, bacteriocytes, and ovarian tissues. The peculiar shape of the root tissues implies that their morphogenesis is regulated by the repeated growth and remodeling of ECMs. Some MMPs may also have a function during the formation of the root tissue.

In vertebrates, cathepsin K (CTSK) is highly expressed in the osteoclast and is responsible for the digestion of type I collagen, which is the main component of mineralized bone [52, 53]. In O. japonicus, we could not find CTSK in the transcriptome, but three $c t s B$ genes were expressed in the root. CTSB is a papain-like cysteine protease that is usually located in the lysozome in normal cells and tissues in humans [54]. In the clam Meretrix meretrix and the shrimp Pandalus borealis, CTSB are expressed in the digestive tract (digestive gland and hepatopancreas, respectively) and have been reported to have a possible role in nutrient digestion $[55,56]$. We showed that some protease genes, mmps and ctsBs, were expressed at the early juvenile stage when the worms start to bore into bones, whereas no expression was detected during the larval stage. The expression patterns of protease genes in the host worm, together with the absence of collagenase genes in Osedax symbionts [27], strengthen the possibility that the root epidermal cells play critical roles in the digestion of bone matrix proteins (Fig. 6).

\section{Nutrient uptake and metabolic interaction between host and symbiotic bacteria}

The SLC superfamily is the largest group of membrane transporter proteins, and includes 52 families and 395 transporter genes in the human genome [33]. The SLC transporters play essential roles in nutrient uptake in the 


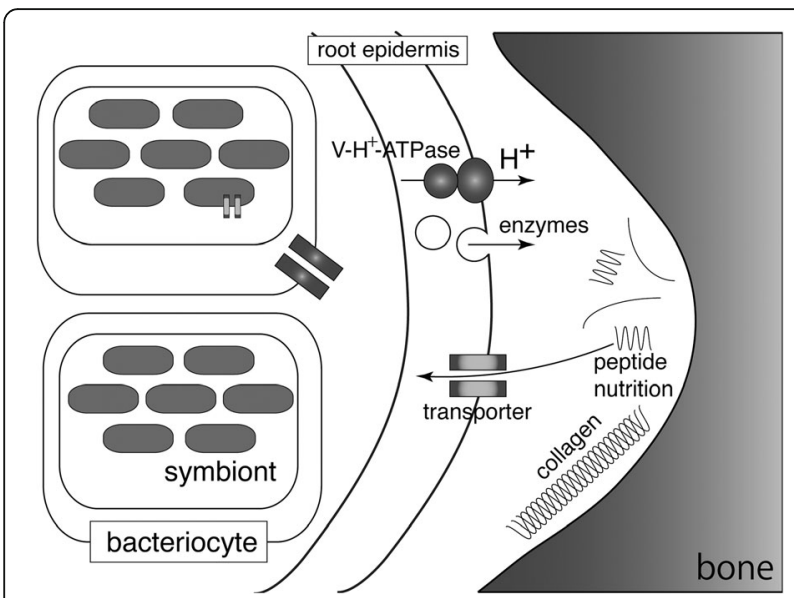

Fig. 6 Schematic illustration of bone digestion and nutrient uptake in Osedax japonicus. Calcium phosphate in vertebrate bone is digested through acidification of the microenvironment by $\mathrm{V}-\mathrm{H}$ ${ }^{+}$-ATPase [24]. Organic components of bones, such as collagens are degraded by proteases secreted from root epidermis. The digested peptides and other nutrients contained in the bones are transported through various membrane transporters. Some of the nutrients are transported to symbiotic bacteria, which use the nutrients as a metabolic resource

intestine as well as in the translocation of various kinds of molecules in other tissues and cell types. The functions of each member of the SLC transporter are well characterized in humans [33]; however, little is known about the function of SLC transporters in invertebrates. Almost all families of the SLC transporters are conserved among bilaterians [57]. In this study, we confirmed the expression of some slc genes encoding oligopeptide and amino acid transporters in the root epidermis of O. japonicus. In mammals, members of the proton-coupled oligopeptide transporter family (SLC15) are responsible for the absorption of dietary protein digestion products in the intestine [34]. In O. japonicas, we found that two genes encoding SLC15 family transporters were expressed in the root epidermis. Root epidermal cells started to express one of two slc15 genes at the early juvenile stage, when the animals began a sessile lifestyle and started consuming vertebrate bones. We also detected expression of a gene encoding the amino acid transporter family (SLC6) in the root epidermis. These transporters are the candidate molecules playing a crucial role in nutrient uptake. Further functional analysis will unveil the molecular mechanisms for digestion and absorption by the root of Osedax worms.

In this study, we found the expression of genes encoding amino acid, glucose, and monocarboxylate transporters in bacteriocytes. The genome sequence of Osedax symbionts showed that they encode a number of transporter genes for various substrates such as amino acids, peptides, and carbohydrates [27]. The exact function of symbiotic bacteria in Osedax worms is still unclear; however, expression and possession of transporter genes in the host bacteriocytes and symbiont genome suggest the presence of metabolic interaction between Osedax and symbiotic bacteria (Fig. 6).

\section{Conclusions}

Because there is no comparable counterpart close relative of Osedax, the root is considered an evolutionary novelty. Here, we investigated tissue-specific transcriptome and the expression pattern of genes, which appear to be related to the function of the root. Expression of various proteinase including $m m p$ and transporter genes in the root epidermis support the theory that the root epidermal cells are responsible for bone digestion and subsequent nutrient uptake. Expression of amino acid, sugar and other metabolite transporter genes in the host bacteriocytes suggests the presence of metabolic interaction between Osedax and symbiotic bacteria. Further studies using the $O$. japonicus and $N$. japonica system, which allows us to examine the biology of both species and the interactions between them, should provide essential information regarding the evolutionary novelty of Osedax.

\section{Methods}

\section{Animal collection and laboratory culture}

We obtained $O$. japonicus specimens from whale bones collected at a depth of $226 \mathrm{~m}$ off Cape Noma Misaki via a remotely operated vehicle (ROV), Hyper-Dolphin, on the research vessel (R/V) Natsushima on March 28 and April 13, 2012. Osedax japonicus specimens were kept in 100-L tanks at $11{ }^{\circ} \mathrm{C}$ in the laboratory together with whale bones. Embryos and males were collected from the mucus of females. Larval settlement was induced by adding small pieces of vertebrate bone to dishes where the larvae were kept [28]. To induce the infection of $N$. japonica, we incubated the bones with $N$. japonicus overnight at $20{ }^{\circ} \mathrm{C}$. After a brief wash with filtered artificial sea water, the bones were cultured with larvae.

\section{RNA extraction and sequencing}

We extracted total RNA from three samples: the female trunk + palps (trunk), the root + ovisac (root), and larvae + adult males, using QIAzol reagent followed by column purification using RNeasy Mini Kit (Qiagen, Limburg, Netherlands) according to the manufacturer's instructions. The quality and concentration of total RNA was analyzed by gel electrophoresis and a NanoDrop Spectrophotometer (Agilent, Santa Clara, CA, USA), respectively. Only samples that met the manufacturer's criteria (BGI: http:// www.bgi.com/us/services/genomics/whole-transcriptomesequencing/) were then processed for the RNA-seq analysis. Short read sequences were obtained using a 
Hiseq2000 sequencer (Illumina, San Diego, CA, USA) according to the manufacturer's procedures. High quality bases (quality score $\geq 20$ ) were used in following analyses. The raw sequence data are available in the DDBJ sequence Read Archive (DRA003880).

\section{Bioinformatic analyses}

Three fastq files obtained from the samples were combined into a dataset and de novo assembly of the O. japonicus transcriptome without a reference genome was performed using Trinity (version r2013-02-25) [29]. To obtain normalized gene expression intensities (in FPKM), reads from each of the three samples were mapped onto the Trinity assembly with the bowtie [58], and analyzed with the RNA-seq by Expectation-Maximization (RSEM: version 1.2.3; [59]) and edgeR [60]. In the assembly procedure, putative alternative splicing variants were estimated as different contigs per sub-component; however we merged variants from one sub-component based on the "\%comp_fpkm" values of the RSEM output when we estimated the tissue specificity of each sub-component. The Maser analytical pipelines on the National Institute of Genetics Cell Innovation program (http://cell-innovation. nig.ac.jp/) were used for the following functional estimations of the assembled Trinity contigs.

$\mathrm{GO}$ enrichment analysis was employed to identify GO terms associated with a subset of genes and to test whether this association (enrichment) is significantly different from what would be expected by chance given a background gene set (in this case, the entire gene set). GO terms were assigned to nucleotide contigs of the Osedax assembly against a set of the protein database of UniprotKB that showed BLASTX hits with a threshold E-value of $10^{-10}$. FatiGo was employed for the statistical enrichment test of root specific genes against the pool of all transcriptome based on the annotated subcomponents after a false discovery rate $<0.01$ correction [61].

For the protein annotation analyses of Osedax sequences, the longest open reading frames and corresponding amino acid sequences per subcomponents were predicted using a Perl script, with sequences longer than 30 amino acids used for downstream analysis. Transmembrane domains and signal sequence regions were predicted with TMHMM 2.0 [62] and SignalP 4.1 [63]. Pfam domains were assigned based on the results of a HMMER (3.1b2; [64]) search against the Pfam-A database [30], with a threshold E-value of $10^{-5}$. The statistical analyses of the Pfam enrichment analysis were performed using $\mathrm{R}$ (3.1.2), with the Q-value package (1.43.0; [65]). The $p$-values of the enriched domains in the root-specific gene set against the pool of three transcriptomes were calculated using a hypergeometric distribution, with a false discovery rate $<0.05$ correction. The Pfam enrichment analyses were performed using domains that were assigned to at least five subcomponents.

\section{Phylogenetic analyses of genes}

For the analysis counterpart of Osedax, we searched for mmp genes from three spiralians genomes already published: the pacific oyster Crassostrea gigas [48], the owl limpet Lottia gigantea, and a marine polychaete Capitella teleta [49], using BLAST on the genome browsers of the animals. We first performed TBLASTN against each genome using Osedax MMP sequences as queries (an E-value of $<1 \mathrm{e}^{-10}$ ). Gene orthologies were confirmed by the phylogenetic analyses described later.

Fragments of genes were amplified by PCR (the primer sequences and accession numbers are in Additional file 9: Table S6). Gene orthologies were inferred by maximum likelihood analyses and multiple alignments. Amino-acid alignments were made with MAFFT ver. 7 [66]. Sequences were trimmed by trimAl [67]. Maximum likelihood analyses were performed with RAxML ver. 8 [68].

\section{In situ hybridization}

Animals were fixed with $4 \%$ paraformaldehyde (PFA) in Mops buffer $(0.1 \mathrm{M}$ Mops, $0.5 \mathrm{M} \mathrm{NaCl})$ at $4{ }^{\circ} \mathrm{C}$, overnight. After several washes with PBST (i.e., PBS containing 0.1\% Tween 20), samples were dehydrated through an ethanol series and stored in $80 \%$ ethanol at $-20{ }^{\circ} \mathrm{C}$. We used two in situ hybridization methods for visualizing spatiotemporal gene expression patterns. For the whole-mount in situ hybridization of larvae and juvenile females, samples were rehydrated and washed three times with PBST. The samples were digested with $1 \mu \mathrm{g} / \mathrm{ml}$ proteinase K/PBST for 20 min at $37^{\circ} \mathrm{C}$. After a brief wash with PBST, the samples were postfixed in $4 \% \mathrm{PFA} / \mathrm{PBST}$ for $10 \mathrm{~min}$ at room temperature $\left(20-25{ }^{\circ} \mathrm{C}, \mathrm{RT}\right)$, and washed three times with PBST. The samples were prehybridized in hybridization solution $(50 \%$ formamide, $5 \times$ SSC, $5 \times$ Denhardt's solution, $100 \mu \mathrm{g} / \mathrm{ml}$ yeast RNA, and $0.1 \%$ Tween 20 ) at $55{ }^{\circ} \mathrm{C}$ for $2 \mathrm{~h}$ and hybridized with a hybridization solution containing a digoxigenin (DIG)-labeled RNA probe at $55^{\circ} \mathrm{C}$ for at least $16 \mathrm{~h}$. For the negative control, an RNA probe of a gene, which was not expressed in the stages examined, was used (data not shown). The samples were washed with a solution of $50 \%$ formamide, $4 \times$ SSC, and $0.1 \%$ Tween 20 for 30 min twice; $50 \%$ formamide, $2 \times$ SSC, and $0.1 \%$ Tween 20 for 30 min twice; $2 \times$ SSC and $0.1 \%$ Tween 20 for 30 min twice; and $0.2 \times$ SSC and $0.1 \%$ Tween 20 for $30 \mathrm{~min}$ twice at $55{ }^{\circ} \mathrm{C}$. The samples were washed with MABT (i.e., maleic acid buffer containing $0.1 \%$ Tween 20 ) three times, blocked in $2 \%$ blocking reagent (Roche, Indianapolis, IN, USA) in MABT for $60 \mathrm{~min}$ at RT, and incubated overnight at $4{ }^{\circ} \mathrm{C}$ with a 1:1500 dilution of antiDIG-AP antibody (Roche) in the blocking buffer. Samples were then washed six times with MABT for $60 \mathrm{~min}$ and transferred into AP buffer (100 mM Tris pH 9.5, $100 \mathrm{mM}$ $\mathrm{NaCl}, 50 \mathrm{mM} \mathrm{MgCl} 2$, and $10 \%$ dimethylformamide) without $\mathrm{MgCl}_{2}$ and dimethylformamide. After being washed 
twice with AP buffer, a chromogenic reaction was performed using nitro blue tetrazolium chloride/5-bromo-4chloro-3-indolyl-phosphate (NBT/BCIP; Roche) in AP buffer until signals were visible. The reaction was stopped in PBST, postfixed in 4\% PFA/PBST, rewashed with PBST, and mounted with $40 \%$ glycerol before being observed under a light microscope (IX71, Olympus).

For frozen sections, bones containing adult $O$. japonicas females were fixed overnight with $4 \%$ PFA/MOPS at $4{ }^{\circ} \mathrm{C}$. The fixed samples were washed three times with PBS. We digested bones overnight with Morse's solution at RT [69]. After we had picked worms from the bones, they were washed three times with PBS and mounted in Tissue-Tek ${ }^{\circ}$ O.C.T compound to use as frozen sections. The in situ hybridization protocol of the frozen sections of adult females was based on a previous report [70]. For double staining, we used a fluorescein-labeled probe for hybridization. After the chromogenic reaction with NBT/ BCIP described above, slides were incubated in $0.1 \mathrm{M}$ glycine- $\mathrm{HCl}$ for $30 \mathrm{~min}$ to inactivate the alkaline phosphatase. Slides were washed three times with $M A B$, blocked in 2\% blocking reagent in MAB for $60 \mathrm{~min}$ at RT, and incubated for $1 \mathrm{~h}$ at RT with a 1:2000 dilution of antifluorescein-AP antibody (Roche) in blocking buffer. They were washed six times with MAB for 60 min and transferred into AP buffer without $\mathrm{MgCl}_{2}$. After being washed twice with AP buffer, a chromogenic reaction was performed with 2-(4-iodophynyl)-5-(4-nitrophenyl)-3-pheniltetrazolium chloride/5-bromo-4-chloro-3-indolyl-phosphate (INT/BCIP; Roche) in AP buffer until signals were visible. The reaction was stopped in PBST, postfixed in $4 \%$ PFA/PBS, rewashed with PBS, and mounted with $80 \%$ glycerol. The slides were then observed under a light microscope.

\section{Additional files}

Additional file 1: Figure S1. Gene expression intensity (fragments per kilobase per million reads) distribution of the Osedax japonicus transcriptome. (PDF $242 \mathrm{~kb}$ )

Additional file 2: Table S1. Summary of the transcriptome analysis. (XLSX $41 \mathrm{~kb}$ )

Additional file 3: Figure S2. Scatter plots of Osedax japonicus transcriptome. Root specific genes are indicated as magenta plots. Gray plots in the root specific area ( $X$-axis $<0, Y$-axis $\geq 0$ ) are genes whose fragments per kilobase per million reads (FPKM) values of the other sample were above the threshold $(\geq 0)$. For example, gray plots in the root specific area of the left graph are genes whose trunk FPKM values were above the threshold. (PDF $451 \mathrm{~kb}$ )

Additional file 4: Table S2. List of the root specific transcriptome. (XLSX $61 \mathrm{~kb}$ )

Additional file 5: Table S3. Significantly enriched gene ontology (GO) terms in the root transcriptome. (XLSX $45 \mathrm{~kb}$ )

Additional file 6: Table S4. Summary of $\mathrm{mmp}$ gene expression. (XLSX $47 \mathrm{~kb})$
Additional file 7: Figure S3. Phylogenetic trees of genes. The numbers at the nodes are bootstrap values (only those $\geq 50 \%$ are shown). Ac, Aplysia californica; Bb, Branchiostoma belcheri; $\mathrm{Bf}$, Branchiostoma floridae: Cg, Crassostrea gigas; Ci, Ciona intestinalis; Ct, Capitella teleta; Dm, Drosophila melanogaster; Hs, Homo sapiens; Lg, Lottia gigantea; Mm, Mus musculus; Nv, Nematostella vectensis; Oj, Osedax japonicus; Pf, Pinctada fucata; Sk, Saccoglossus kowalevskii; Sp, Strongylocentrotus purpurtus; Tc, Tribolium castaneum. (PDF 528 kb)

Additional file 8: Table S5. Solute carrier (SLC) transporters in the Osedax japonicus transcriptome. (XLSX $31 \mathrm{~kb}$ )

Additional file 9: Table S6. Primer sets and accession numbers of genes. (XLSX $53 \mathrm{~kb}$ )

\section{Acknowledgement}

We are grateful to the captain and crew of the R/ Natsushima and the operation team of the ROV Hyper-Dolphin for animal collection. We thank to Hiroshi Wada for his help in informatics analysis, and Michiko Yano for her help in the molecular biological experiments. The authors utilized the NIG Cell Innovation program (http://cell-innovation.nig.ac.jp/index_en.html) for the informatics analysis. This work is supported by Grants-in-Aid for Research Activity

Start-up 23870044 to NM.

\section{Availability of data and materials}

Raw short read sequences are available in the DDBJ Sequence Read Archive under the accession number DRA003880.

\section{Authors' contributions}

NM conceived the project, conducted the experiments, and drafted the manuscript. MAY conceived the project, performed the bioinformatic analysis, and drafted the manuscript. HK performed the bioinformatic analysis and drafted the manuscript. YF supervised the project. All authors have read and approved the final manuscript.

\section{Competing interests}

The authors declare that they have no competing interests.

\section{Consent for publication}

Not applicable.

Ethics approvals and consent to participate

Not applicable.

\section{Author details}

'Japan Agency for Marine-Earth Science and Techonology, Yokosuka, Kanagawa, Japan. ${ }^{2}$ National Institute of Genetics, Mishima, Shizuoka, Japan. ${ }^{3}$ Postodoctral research fellow, Japanese Society for Promotion of Science, Tokyo, Japan. ${ }^{4}$ Graduate School of Life and Environmental Sciences, University of Tsukuba, Tsukuba, Ibaraki, Japan.

Received: 29 April 2016 Accepted: 6 December 2016 Published online: 13 January 2017

References

1. Corliss JB, Dymond J, Gordon LI, Edmond JM, von Herzen RP, Ballard RD, et al. Submarine thermal sprirngs on the galapagos rift. Science. 1979;203:1073-83.

2. German CR, Ramirez-Llodra E, Baker MC, Tyler PA. ChEss Scientific Steering C: Deep-water chemosynthetic ecosystem research during the census of marine life decade and beyond: a proposed deep-ocean road map. PLOS ONE. 2011;6:e23259.

3. Van Dover CL, German CR, Speer KG, Parson LM, Vrijenhoek RC. Evolution and biogeography of deep-sea vent and seep invertebrates. Science. 2002;295:1253-7.

4. Vrijenhoek R. Genetics and evolution of deep-sea chemosynthetic bacteria and their invertebrate hosts. In: Kiel S, editor. The Vent and Seep Biota Topics in Geobiology, vol. 33. Berlin: Springer; 2010. p. 15-49.

5. Dubilier N, Bergin C, Lott C. Symbiotic diversity in marine animals: the art of harnessing chemosynthesis. Nat Rev Microbiol. 2008;6:725-40.

6. Smith $C R$, Kukert $H$, Wheatcroft RA, Jumars PA, Deming JW. Vent fauna on whale remains. Nature. 1989;341:27-8. 
7. Smith CR, Baco AR. Ecology of whale falls at the deep-sea floor. Oceanogr Mar Biol Annu Rev. 2003;41:311-54.

8. Rouse GW, Goffredi SK, Vrijenhoek RC. Osedax: bone-eating marine worms with dwarf males. Science. 2004;305:668-71.

9. Jones WJ, Johnson SB, Rouse GW, Vrijenhoek RC. Marine worms (genus Osedax) colonize cow bones. Proc R Soc B. 2008;275:387-91.

10. Rouse GW, Goffredi SK, Johnson SB, Vrijenhoek RC. Not whale-fall specialists, Osedax worms also consume fishbones. Biol Lett. 2011;7:736-9.

11. Rouse GW, Wilson NG, Worsaae K, Vrijenhoek RC. A dwarf male reversal in bone-eating worms. Curr Biol. 2015;25:236-41.

12. Goffredi SK, Paull CK, Fulton-Bennett K, Hurtado LA, Vrijenhoek RC. Unusua benthic fauna associated with a whale fall in Monterey Canyon, California. Deep Sea Res I. 2004;51:1295-306.

13. Glover AG, Kallstrom B, Smith CR, Dahlgren TG. World-wide whale worms? A new species of Osedax from the shallow north Atlantic. Proc R Soc B. 2005;272:2587-92.

14. Fujikura K, Fujiwara $Y$, Kawato M. A new species of Osedax (Annelida: Siboglinidae) associated with whale carcasses off Kyushu, Japan. Zoolog Sci. 2006;23:733-40.

15. Braby CE, Rouse GW, Johnson SB, Jones WJ, Vrijenhoek RC. Bathymetric and temporal variation among Osedax boneworms and associated megafauna on whale-falls in Monterey Bay, California. Deep Sea Res I. 2007;54:1773-91.

16. Vrijenhoek RC, Johnson SB, Rouse GW. A remarkable diversity of boneeating worms (Osedax; Siboglinidae; Annelida). BMC Biol. 2009;7:74.

17. Salathe RM, Vrijenhoek RC. Temporal variation and lack of host specificity among bacterial endosymbionts of Osedax bone worms (Polychaeta: Siboglinidae). BMC Evol Biol. 2012;12:189.

18. Rouse GW, Worsaae K, Johnson SB, Jones WJ, Vrijenhoek RC. Acquisition of dwarf male "Harems" by recently settled females of Osedax roseus n. sp (Siboglinidae; Annelida). Biol Bull. 2008;214:67-82.

19. Glover AG, Wiklund H, Taboada S, Avila C, Cristobo J, Smith CR, et al. Bone-eating worms from the Antarctic: the contrasting fate of whale and wood remains on the Southern Ocean seafloor. Proc R Soc B. 2013;280:20131390.

20. Goffredi SK, Orphan VJ, Rouse GW, Jahnke L, Embaye T, Turk K, et al. Evolutionary innovation: a bone-eating marine symbiosis. Environ Microbiol. 2005:7:1369-78

21. Hall BK, Kerney R. Levels of biological organization and the origin of novelty. J Exp Zool B Mol Dev Evol. 2012;318:428-37.

22. Higgs ND, Glover AG, Dahlgren TG, Little CTS. Bone-boring worms: characterizing the morphology, rate, and method of bioerosion by Osedax mucofloris (Annelida, Siboglinidae). Biol Bull. 2011;221:307-16.

23. Goffredi SK, Johnson SB, Vrijenhoek RC. Genetic diversity and potential function of microbial symbionts associated with newly discovered species of Osedax polychaete worms. Appl Environ Microbiol. 2007;73:2314-23.

24. Tresguerres M, Katz S, Rouse GW. How to get into bones: proton pump and carbonic anhydrase in Osedax boneworms. Proc R Soc B. 2013;280:20130625.

25. Katz S, Klepal W, Bright M. The skin of Osedax (Siboglinidae, Annelida): an ultrastructural investigation of its epidermis. J Morphol. 2010;271:1272-80.

26. Katz S, Klepal W, Bright M. The Osedax Trophosome: Organization and Ultrastructure. Biol Bull. 2011;220:128-39.

27. Goffredi SK, Yi H, Zhang Q, Klann JE, Struve IA, Vrijenhoek RC, Brown CT. Genomic versatility and functional variation between two dominant heterotrophic symbionts of deep-sea Osedax worms. ISME J. 2014;8:908-24.

28. Miyamoto N, Yamamoto T, Yusa Y, Fujiwara Y. Postembryonic development of the bone-eating worm Osedax japonicus. Naturwissenschaften. 2013:100:285-9.

29. Grabherr MG, Haas BJ, Yassour M, Levin JZ, Thompson DA, Amit I, et al. Full-length transcriptome assembly from RNA-Seq data without a reference genome. Nat Biotechnol. 2011;29:644-52.

30. Finn RD, Bateman A, Clements J, Coggill P, Eberhardt RY, Eddy SR, et al. Pfam: the protein families database. Nucleic Acids Res. 2014;42:D222-230.

31. Katz S, Rouse GW. The reproductive system of Osedax (Annelida, Siboglinidae): ovary structure, sperm ultrastructure, and fertilization mode. Invertebr Biol. 2013;132:368-85.

32. He L, Vasiliou K, Nebert DW. Analysis and update of the human solute carrier (SLC) gene superfamily. Hum genomics. 2009;3:195-206.

33. Hediger MA, Clemencon B, Burrier RE, Bruford EA. The ABCs of membrane transporters in health and disease (SLC series): introduction. Mol Aspects Med. 2013;34:95-107.

34. Smith DE, Clemencon B, Hediger MA. Proton-coupled oligopeptide transporter family SLC15: physiological, pharmacological and pathological implications. Mol Aspects Med. 2013;34:323-36.
35. Pramod AB, Foster J, Carvelli L, Henry LK. SLC6 transporters: structure, function, regulation, disease association and therapeutics. Mol Aspects Med. 2013;34:197-219.

36. Fotiadis D, Kanai Y, Palacin M. The SLC3 and SLC7 families of amino acid transporters. Mol Aspects Med. 2013;34:139-58.

37. Reimer RJ. SLC17: a functionally diverse family of organic anion transporters. Mol Aspects Med. 2013;34:350-9.

38. Wright EM. Glucose transport families SLC5 and SLC50. Mol Aspects Med. 2013;34:183-96.

39. Halestrap AP. The SLC16 gene family - structure, role and regulation in health and disease. Mol Aspects Med. 2013;34:337-49.

40. Bergeron MJ, Clemencon B, Hediger MA, Markovich D. SLC13 family of $\mathrm{Na}(+)$-coupled di- and tri-carboxylate/sulfate transporters. Mol Aspects Med. 2013;34:299-312.

41. Romero MF, Chen AP, Parker MD, Boron WF. The SLC4 family of bicarbonate $\left(\mathrm{HCO}_{3}^{-}\right)$transporters. Mol Aspects Med. 2013;34:159-82.

42. Blair HC, Teitelbaum SL, Ghiselli R, Gluck S. Osteoclastic bone resorption by a polarized vacuolar proton pump. Science. 1989;245:855-7.

43. Vu TH, Werb Z. Matrix metalloproteinases: effectors of development and normal physiology. Genes Dev. 2000;14:2123-33.

44. Wada H. Origin and genetic evolution of the vertebrate skeleton. Zoolog Sci. 2010;27:119-23.

45. Huxley-Jones J, Clarke TK, Beck C, Toubaris G, Robertson DL, Boot-Handford RP. The evolution of the vertebrate metzincins; insights from Ciona intestinalis and Danio rerio. BMC Evol Biol. 2007;7:63.

46. Angerer L, Hussain S, Wei Z, Livingston BT. Sea urchin metalloproteases: a genomic survey of the BMP-1/tolloid-like, MMP and ADAM families. Dev Biol. 2006:300:267-81.

47. Fanjul-Fernandez M, Folgueras AR, Cabrera S, Lopez-Otin C. Matrix metalloproteinases: evolution, gene regulation and functional analysis in mouse models. Biochim Biophys Acta. 1803;2010:3-19.

48. Ortega N, Behonick DJ, Werb Z. Matrix remodeling during endochondral ossification. Trends Cell Biol. 2004;14:86-93.

49. Simakov O, Marletaz F, Cho SJ, Edsinger-Gonzales E, Havlak P, Hellsten U, et al. Insights into bilaterian evolution from three spiralian genomes. Nature. 2013:493:526-31.

50. Knorr E, Schmidtberg H, Vilcinskas A, Altincicek B. MMPs regulate both development and immunity in the tribolium model insect. PLoS ONE. 2009;:4:e4751.

51. Deady LD, Shen W, Mosure SA, Spradling AC, Sun J. Matrix metalloproteinase 2 is required for ovulation and corpus luteum formation in Drosophila. PLoS Genet. 2015;11:e1004989.

52. Garnero P, Borel O, Byrjalsen I, Ferreras M, Drake FH, McQueney MS, et al. The collagenolytic activity of cathepsin $\mathrm{K}$ is unique among mammalian proteinases. J Biol Chem. 1998;273:32347-52.

53. Inaoka T, Bilbe $G$, Ishibashi O, Tezuka K, Kumegawa M, Kokubo T. Molecular cloning of human cDNA for cathepsin K: novel cysteine proteinase predominantly expressed in bone. Biochem Biophys Res Commun. 1995;206:89-96.

54. Baici A, Horler D, Lang A, Merlin C, Kissling R. Cathepsin B in osteoarthritis: zonal variation of enzyme activity in human femoral head cartilage. Ann Rheum Dis. 1995;54:281-8.

55. Aoki H, Ahsan MN, Watabe S. Molecular cloning and characterization of cathepsin B from the hepatopancreas of northern shrimp Pandalus borealis. Comp Biochem Physiol B Biochem Mol Biol. 2003;134:681-94.

56. Yao X, Zhang J, Sun J, Liu B. Recombinant expression, characterization and expressional analysis of clam Meretrix meretrix cathepsin B, an enzyme involved in nutrient digestion. Mol Biol Rep. 2011;38:1861-8.

57. Hoglund PJ, Nordstrom KJ, Schioth HB, Fredriksson R. The solute carrier families have a remarkably long evolutionary history with the majority of the human families present before divergence of Bilaterian species. Mol Biol Evol. 2011;28:1531-41.

58. Langmead B, Trapnell C, Pop M, Salzberg SL. Ultrafast and memory-efficient alignment of short DNA sequences to the human genome. Genome Biol. 2009;10:R25

59. Li B, Dewey CN. RSEM: accurate transcript quantification from RNA-Seq data with or without a reference genome. BMC Bioinformatics. 2011;12:323.

60. Robinson MD, McCarthy DJ, Smyth GK. edgeR: a Bioconductor package for differential expression analysis of digital gene expression data. Bioinformatics. 2010;26:139-40. 
61. Al-Shahrour F, Diaz-Uriarte R, Dopazo J. FatiGO: a web tool for finding significant associations of Gene Ontology terms with groups of genes. Bioinformatics. 2004;20:578-80.

62. Krogh A, Larsson B, von Heijne G, Sonnhammer EL. Predicting transmembrane protein topology with a hidden Markov model: application to complete genomes. J Mol Biol. 2001;305:567-80.

63. Petersen TN, Brunak S, von Heijne G, Nielsen H. SignalP 4.0: discriminating signal peptides from transmembrane regions. Nat Methods. 2011;8:785-6.

64. Eddy SR. Profile hidden Markov models. Bioinformatics. 1998;14:755-63.

65. Storey JD with contributions from Bass AJ, Dabney A, Robinson D. qvalue: Q-value estimation for false discovery rate control. R package version 2.4.2. 2015. http://github.com/jdstorey/qvalue.

66. Katoh K, Standley DM. MAFFT multiple sequence alignment software version 7: improvements in performance and usability. Mol Biol Evol. 2013;30:772-80.

67. Capella-Gutierrez S, Silla-Martinez JM, Gabaldon T. trimAl: a tool for automated alignment trimming in large-scale phylogenetic analyses Bioinformatics. 2009;25:1972-3.

68. Stamatakis A. RAxML version 8: a tool for phylogenetic analysis and post-analysis of large phylogenies. Bioinformatics. 2014;30:1312-3.

69. Shibata Y, Fujita S, Takahashi H, Yamaguchi A, Koji T. Assessment of decalcifying protocols for detection of specific RNA by non-radioactive in situ hybridization in calcified tissues. Histochem Cell Biol. 2000;113:153-9.

70. Miyamoto $\mathrm{N}$, Wada $\mathrm{H}$. Hemichordate neurulation and the origin of the neural tube. Nat commun. 2013;4:2713.

\section{Submit your next manuscript to BioMed Central and we will help you at every step:}

- We accept pre-submission inquiries

- Our selector tool helps you to find the most relevant journal

- We provide round the clock customer support

- Convenient online submission

- Thorough peer review

- Inclusion in PubMed and all major indexing services

- Maximum visibility for your research

Submit your manuscript at www.biomedcentral.com/submit

) Biomed Central 